\title{
Mechanically Twisting of Multi-Membrane Carbon Nanotube Yarn and the Enhancement of Its Mechanical Properties
}

\author{
Zhu Jingdong* \\ School of Materials Science and Engineering, Tianjin University, Weijin Road 92, Nankai District, \\ Tianjin 300072, P. R. China. \\ *Corresponding Author: Doctor Zhu Jingdong Email: zhujing_dong@126.com
}

Keywords: Carbon Nanotube Fibers, Twisting, Tensile Strength, Modulus

\begin{abstract}
The direct CVD-spun multi-membrane carbon nanotube yarn is mechanically twisted to engineer its structures and to enhance its mechanical properties. Homogenously twisted CNT yarns with a dense cross-section are achieved with different degrees of twisting. The mechanical twisting remarkably increases the tensile strength and modulus of the yarn as the result of radial compressive stress. At a twisting degree of $70 \mathrm{r} / \mathrm{cm}$, the tensile strength of the yarn increases by two folds and the modulus by ten folds of those of the as-spun yarn. The corresponding twisting angle is $32^{\circ}$, and the critical twisting coefficient is $32 \operatorname{tex}^{1 / 2} \mathrm{r} / \mathrm{cm}$. As the result of the increases in strength and modulus, the mean fractural toughness of the yarn is increased by two times. The Poisson ratio of the twisted yarn is decreased to $0.17 \sim 0.20$ from that of the as-spun fibers $(0.25 \sim 0.27)$, as the result of twisting induced yarn densification. This study demonstrates the possibility to engineer the structures of the CNT membrane yarn and to enhance their mechanical performance via the mechanical twisting.
\end{abstract}

\section{Introduction}

Twisting is traditionally used in textile industry to spin wools into a thread, to increase the strength of a yarn and to change the elastic properties of a yarn to meet the desired properties for a fiber in weaving of a fabric ${ }^{[1-2]}$. Currently, there is a great interest to the development of strong continuous fibers of carbon nanotubes for their potential applications as strong fibers and multi-functional fabrics. Macroscopic carbon nanotube fibers are assembled by post-processing methods including wet drawing from a CNT dispersion ${ }^{[3]}$ and dry drawing from carbon nanotube arrays on a substrate ${ }^{[4-6]}$, and by directly spinning from the chemical vapor deposition process ${ }^{[7]}$. While there has been a great challenge in the spinning of continuous CNT fiber, essentially continuous CNTFs of length over kilometers have been spun from the CVD reactions by the direct spinning process. The as-spun fibers have the usual multi-membrane hollow structures and are highly electrical conductive which should find important structural and multi-functional applications. This multi-membrane hollow fiber provides alternatives in changing their macroscopic structures to meet their diverse applications including textile fabrics. Another issue is to enhance the mechanical performance of the fiber. Mechanical twisting should be one of the most direct approach to these purposes, as successfully applied in engineering the structures and enhancement of the performance of conventional textile yarns. In the present work, we report mechanical twisting of the direct spun CNTFs to engineer the fiber structures and enhance their mechanical properties.

The CNTs fibers were spun from a CVD gas-flow reaction using acetone as a carbon source with a water densification process. The fiber was formed by mechanical spinning of a multi-membrane CNT sock from the gas flow followed by water densification. The fiber thus obtained has a multi-membrane structure consisting of seamless CNT membranes concentrically compacted and parallel to the fiber axial. The fiber consists of large-diameter (6 - $8 \mathrm{~nm})$ collapsed double-walled CNTs stacked in bundle of $\sim 50 \mathrm{~nm}$ in thickness. The as-spun yarn has a hollow flatten cross-section with the long edge of $168 \mu \mathrm{m}$ and the short edge of $40 \mu \mathrm{m}$ (Figure 1).

The CNTFs used for twisting were from one single filaments of the as-spun yarn for ensuring their structural uniformities. The filament was cut into in $3 \mathrm{~cm}$ pieces and they were twisted using a 
home-made twisting machine with controlled number of turns. They were twisted individually at different number of turns between 90 and 300 turns with 30 intervals, corresponding to a turn density between 30 and $100 \mathrm{r} / \mathrm{cm}$ with $10 \mathrm{r} / \mathrm{cm}$ intervals.

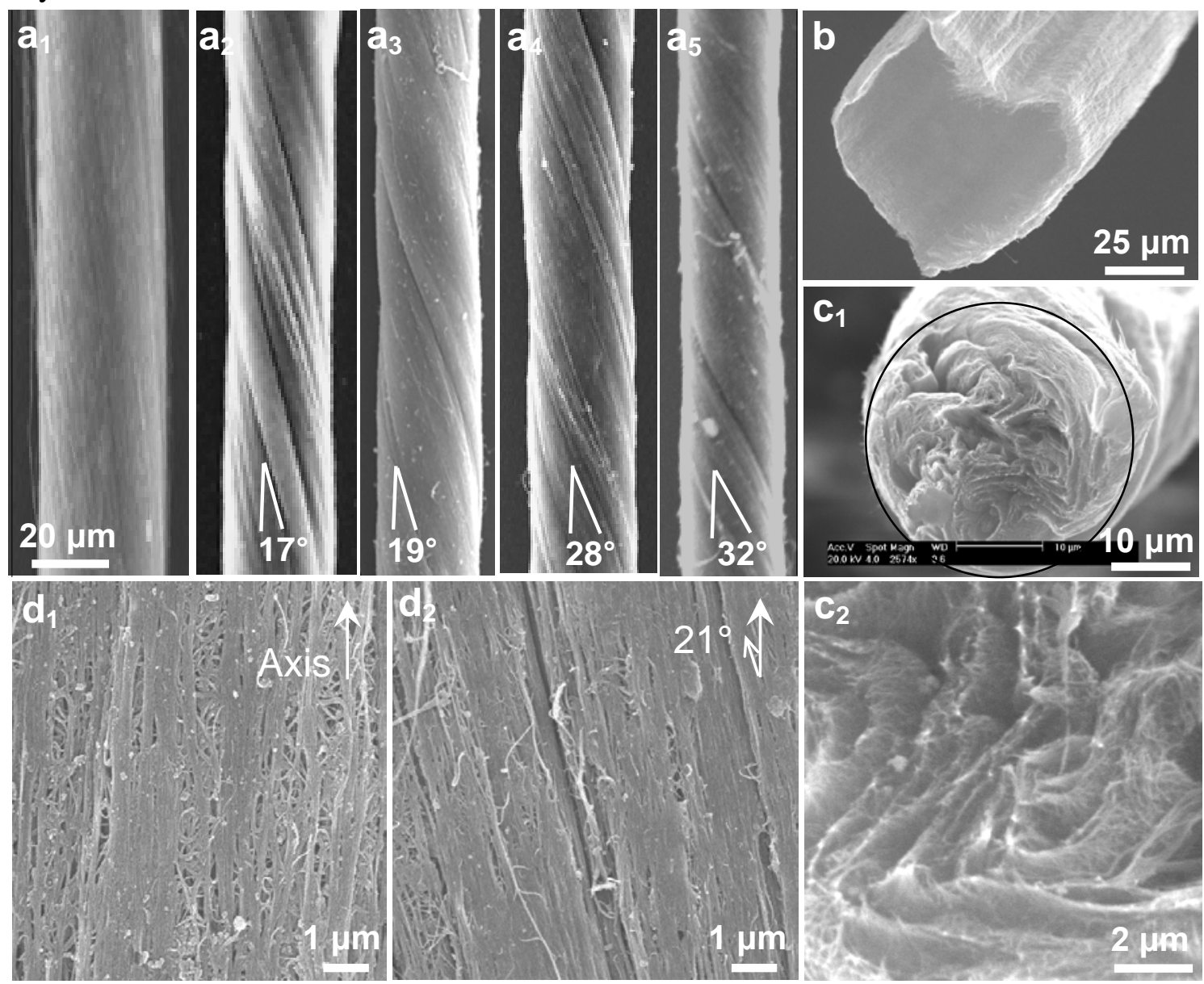

Figure 1 SEM images of overviews of the as-spun membrane fibers and the twisted fibers (a1 - a5), cross-sections (b, c1 - c2) and their surfaces (d1 - d2). From a1-a5, the raw fiber (a1), twisted fibers at $30 \mathrm{r} / \mathrm{cm}$, twisting angle $17^{\circ}$ (a2), 39 $\mathrm{r} / \mathrm{cm}, 19^{\circ}$ (a2), $60 \mathrm{r} / \mathrm{cm}, 28^{\circ}$ (a3) $70 \mathrm{~cm}, 32^{\circ}$. b cross-section of the raw fiber. $\mathrm{c} 1$ and c2, cross-section of a twisted fiber at $70 \mathrm{r} / \mathrm{cm}$, and a local enlargement respectively. $\mathrm{d} 1$ and $\mathrm{d} 2$, the surfaces of the raw fiber and a twisted fiber (70 r/cm) respectively. The twisting angle in a2 - a5 is measured from the SEM images from the tiling of the membranes from the fiber axis. The twisting angle largely represents the tilting of the CNTs within the fibers as seen from the surface of the fibers wherein the CNTs within the fibers tilted with the membranes.The cross-sections are obtained by electrical cutting of the fibers which did not deform the fibers. The electrical cutting was performed by $24 \mathrm{~V}$ voltage. The raw fiber has the wall thickness of $\sim 1 \mu \mathrm{m}$ and outer diameter of $\sim 80 \mu \mathrm{m}$.

The multi-membrane fibers accommodate the mechanical twisting to form a twisted fiber with different degrees of twisting between $30 \mathrm{r} / \mathrm{cm}$ and $100 \mathrm{r} / \mathrm{cm}$. The twisting occurred via the uniform twisting of the entire membranes which just resembles the twisting of a paper roll. Scanning electronic microscopy (SEM) observation shows that the membranes were uniformly twisted without knots and aggregation of twisting on the fiber surface (Figure 1). The twisting angle changes from $17^{\circ}$ to $39^{\circ}$ at twisting between 30 and $100 \mathrm{r} / \mathrm{cm}$ (Figure 1), measured from the SEM images of the twisted fibers. The maximum turns that can be accommodated in the fiber is $100 \mathrm{r} / \mathrm{cm}$. Twisting beyond this value did not cause breaking of the fiber up to $110 \mathrm{r} / \mathrm{cm}$, instead, the extra turns reverted back after the release of one end of the fiber. This indicates the robustness and high flexibility of the multi-membrane fibers.

In contrast, to the hollow flattened walls of the as-spun fiber (Figure b), the twisted fibers become a densified in their cross-section with a round shape, as the result of twisting of the membrane walls (Figure 1). The twisting-induced densifications of the fibers occurred gradually with the degree of twisting between $30-70 \mathrm{r} / \mathrm{cm}$, attaining saturated at $70 \mathrm{r} / \mathrm{cm}$, beyond which no further reduction of the fiber diameter was observed under optical microscopy. From Figure 1, it is 
seen that the apparent diameter of the fiber decreases with the degree of twisting between 30 - 70 $\mathrm{r} / \mathrm{cm}$. At the twisting of $70 \mathrm{r} / \mathrm{cm}$, the apparent diameters of the fiber reduced from $168 \mu \mathrm{m}$ of the as-spun fiber to $30 \mu \mathrm{m}$, reduced by five folds. The SEM view of the cross-section of the twisted fiber shows it consists of twisted membranes (Figure 1). Despite of the densification at the macroscopic scale, the enlarged view of the cross-sections can find the originally compacted multi-membrane walls become separated as the result of shares. On the other hand, SEM observation of the twisted fiber surface shows the CNTs and their bundles became closer packed with reduced voids and spaces among the CNTs in comparing to that of the as-spun fiber (Figure 1 $\mathrm{d} 1, \mathrm{~d} 2$ ). This indicates that the twisting also led to the densification of the CNTs at microscopic scales in-plane of the membranes.

The distinct changes of the fiber structures both at the macroscopic and microscopic scales by twisting lead to great changes in their mechanical performance. Tensile tests show the twisting remarkably increased the fiber strength comparing to the as-spun fibers. The typical strain-stress curves of two twisted fibers by $60 \mathrm{r} / \mathrm{cm}$ and $70 \mathrm{r} / \mathrm{cm}$ and that of the as-spun fibers are shown in Figure 2. The as-spun fiber typically exhibits a typical two-stage of tensile behavior, with an initial high modulus tensile and then turning into a low modulus tensile. The initial lower modulus tensile was due to the stiffness of the mess in the CNT network within the fibers, while the second stage was caused from the sliding of the CNTs in the fiber after the messes failed ${ }^{[8]}$. After the twisting by $60 \mathrm{r} / \mathrm{cm}$ and $70 \mathrm{r} / \mathrm{cm}$, the stress-strain curve still displays the similar styles of the two-stage of tensile behavior as the as-spun fibers but the curve become steeper across the entire stains. This may indicate that the twisting did not change the properties of the structures of the fibers but greatly strengthened both the mess stiffness and the resistance to intra-tube sliding as the result of densification of the structures.
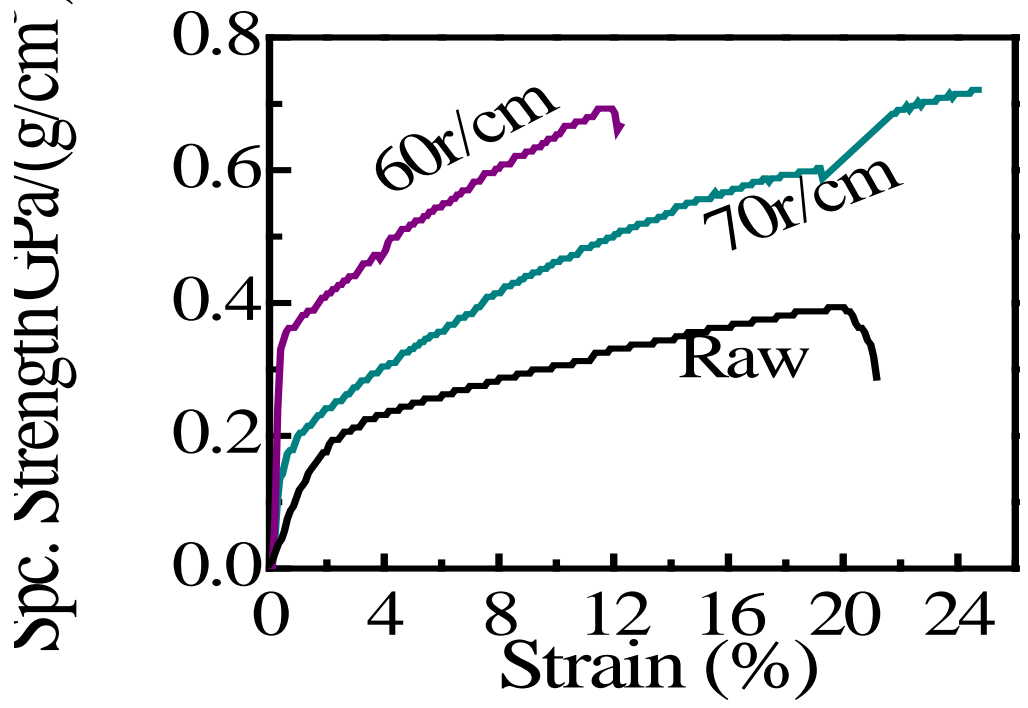

Figure 2 Stress-strain engineer curve of the raw multi-membrane fiber and those after twisting at $60 \mathrm{r} / \mathrm{cm}$ and $70 \mathrm{r} / \mathrm{cm}$ (gauge length $10 \mathrm{~mm}$, and load rate of $3 \mathrm{~mm} / \mathrm{min}$ ).

The fiber strength is evaluated in term of the specific strength $\left(\mathrm{GPa} /\left(\mathrm{g} / \mathrm{cm}^{3}\right)\right.$ which is calculated from the measured force in $\mathrm{N}$ divided by the linear density of the fiber in tex (gram per kilometer fiber, $\mathrm{g} / \mathrm{km}$ ), as $\mathrm{GPa} /\left(\mathrm{g} / \mathrm{cm}^{3}\right)$ is equivalent to $(\mathrm{N} / \mathrm{tex})$. Since the fiber linear density can be precisely measured, the specific strength thus obtained can represent the true strength of the fibers as it does not involve the fiber density in $\mathrm{g} / \mathrm{km}$ which is difficult to be measured. The linear density of the as-spun fiber is 0.32 tex measured by vibration method which gives the linear density for a short fiber by determining the intrinsic vibration of the fibers. After twisting, the fiber tex value did not change distinctly with the changes within $5 \%$. For example, the vibration measurement shows the fiber twisted at $70 \mathrm{r} / \mathrm{cm}$ had a tex value of 0.33 tex, which is $1 \%$ increase. Thus a fixed tex value of 0.32 tex was used for the calculation of the specific strength for all the twisted fibers.

The strength shown is that at the break to failure hence it depends on the elongation of the fibers which may be caused by an incident failure from defects appeared in the fibers subject to tensile. Figure 2 shows that the $60 \mathrm{r} / \mathrm{cm}$ twisted fiber have a higher strength at strain below $12 \%$ 
than the $70 \mathrm{r} / \mathrm{cm}$ twisted fiber, but it fails at this point while the $70 \mathrm{r} / \mathrm{cm}$ did not hence the later giving a higher break-to-failure strength.

Tensile tests of differently twisted fibers show the fiber has been strengthened in the whole range of the fibers being twisted between $30 \mathrm{r} / \mathrm{cm}$ and $100 \mathrm{r} / \mathrm{cm}$ but with different degree of strengthening. The variation of the fiber strength with the twisting angle is plotted in Figure 3. Figure 3 shows that the fiber strength attains maximum at $70 \mathrm{r} / \mathrm{cm}$ and then decreases with the degree of twisting. The specific strength of the twisted fiber is $0.72 \mathrm{GPa} /\left(\mathrm{g} / \mathrm{cm}^{3}\right)$ at $70 \mathrm{r} / \mathrm{cm}$, which is two times higher than that $\left(0.38 \mathrm{GPa} /\left(\mathrm{g} / \mathrm{cm}^{3}\right)\right)$ of the as-spun fiber.

Zhang et al. ${ }^{[6]}$ observed that the dry-spun fiber strength after twisting increases $2.2 \sim 2.4$ times and the modulus increases by $1.2 \sim 2.7$ times and the elongation increase by $3.2 \sim 1.3$ times that of the un-twisted fibers.

The variation of the fiber strength with the twisting angle reflects the general behavior of a twisted fibers made of multi-filaments ${ }^{[9]}$. In general, the increase of strength was due to the friction of the CNTs in the fibers, while as the twisting angle is beyond a certain value, it resulted in sliding of the CNTs thus leading to the reduction of the fiber strength. The twisted degree gives the maximum strength is called the critical angle of twisting. The critical twisting angle for the multi-membrane fiber is $32^{\circ}$, corresponding to $70 \mathrm{r} / \mathrm{cm}$.

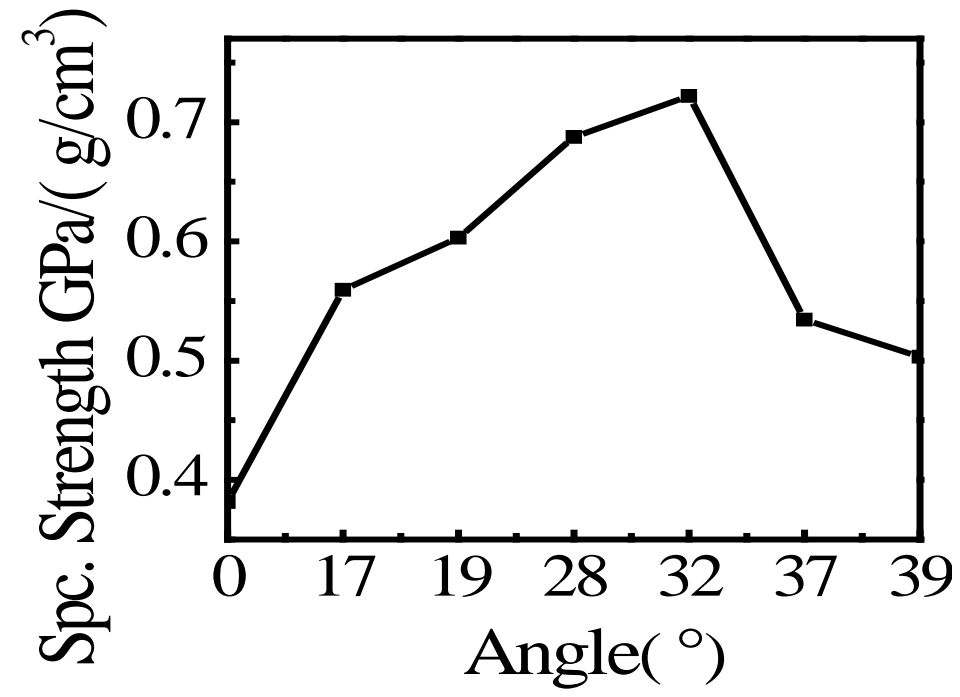

Figure 3 The variation of specific strength of the fibers with the twisting angle.

As the twisting beyond the critical twisting, the outer CNT membranes were severely deformed and resulted in some weak CNT bundles sticking out from the fibers, thus giving reduced tensile strength.

Modulus characterizes the stiffness of a material resistant to stress against deformation, which is characterized by the slope of a strain-stress curve. The tensile curve in Figure 2 indicates that the mechanical twisting remarkably increases the modulus of the fiber in the both regions of tensile. The modulus is the first stage of modulus in the tensile curve (Figure 2). The as-spun fiber has a modulus of $10 \mathrm{GPa}$. After the twisting at $60 \mathrm{r} / \mathrm{cm}$ (twisting angle $28^{\circ}$ ), the modulus of the fiber increased to $100 \mathrm{GPa}$, ten times higher than that of the as-spun fiber (Figure 4). This shows remarkably effects of increasing modulus by twisting. The variation of modulus with twisting angle shows the increases of modulus in the whole range of twisting. The curve shown in Figure 2 shows the modulus increases in the both stages. The modulus in the second stage is increase by two times (from as-spun fiber 1.2 GPa to 3.6 GPa). 


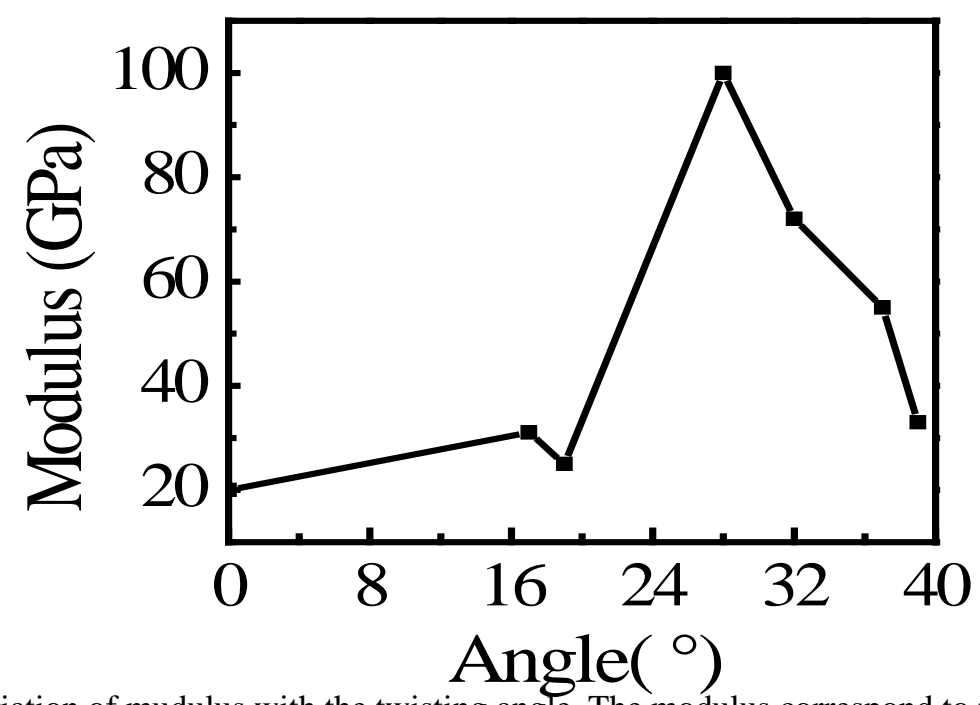

Figure 4 The variation of mudulus with the twisting angle. The modulus correspond to the first stage of tensile in the stress-stress curves as shown in Figure 2.

Twisting may lead to the tilting of the filaments in a fiber from the fiber axial and reduce the fiber stiffness ${ }^{[10]}$. Our CNTFs show different behavior having greatly increased stiffness after twisting. The increase of the modulus was due to the increased friction among the CNT fibers, as the result of the densification of the fibers. The contribution of the inter-membrane friction should be small because the twisted membranes have separated membranes. Especially, the twisting strengthened some weak point in the CNT network.

Zhang et al. found the twisting spun CNTFs have an increased modulus $100 \mathrm{GPa}$ at twisting angle of $21^{\circ}{ }^{[6]}$. They observed the increase of the fiber modulus from 89 to $241 \mathrm{GPa}$ by applying twisting during drawing fiber from a CNT array.

The elongation of the starting fiber is between $10 \%$ and $25 \%$. The maximum elongation may indicate the fully sliding of the CNTs, the elongation attains $25 \%$ for the starting fibers.

The elongation at break point of the fibers after twisting is in the range of $8 \sim 23 \%$, changing irregularly with the degree of twisting (Figure 5). The average elongation is smaller than that of the starting fiber which is $\sim 23 \%$, indicating twisting reduced the elongation. This may indicate the twisting introduced some defects in the fibers as the fiber always breaks at a weakest point. Especially, voids may be increased from the rearrangement of the CNTs within the fibers. Especially, twisting may cause movement of catalyst particles or their aggregation, which may form block barrier for inter-tube sliding. The rearrangement also involves the moving of the catalyst particles, that may be the determinate source of the defects within the fiber. Literature reports an increase of elongation of fiber after twisting ${ }^{[9]}$.

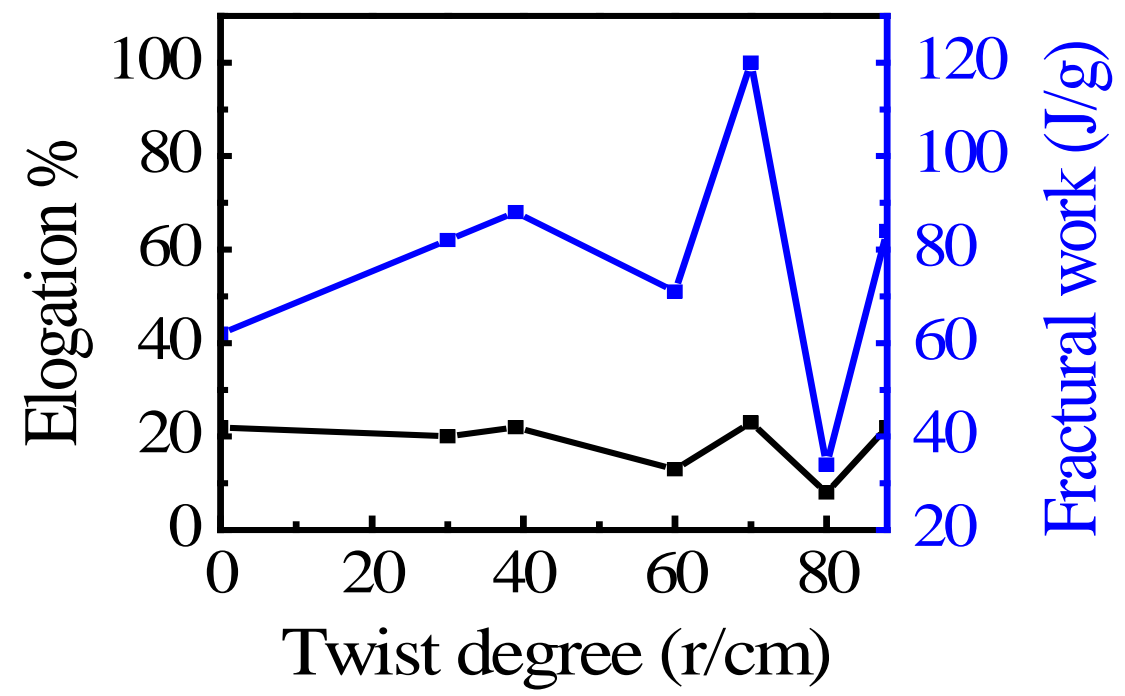

Figure 5 The variation of elongation and the fractural work at the breaking point with the twisting degree of the fiber.

The fractural work of the fiber is represented by the area under the stress-strain curve which 
measures the energy absorbed by the fibers from the pulling before breaking. Despite of the reduced elongation, the increased fiber strength by twisting still gave a noticeable increase of the fractural work by twisting (Figure 5). The fractural work of the fiber after twisting at $70 \mathrm{r} / \mathrm{cm}$ is $120 \mathrm{~J} / \mathrm{g}$, which is double that of the starting fibers which is $63 \mathrm{~J} / \mathrm{g}$. This indicates that twisting also increases the toughness of the fiber. The fractural work of the fiber changes irregularly, since it depends on the elongation which changes irregularly. In comparing the result, the maximum fractural work attains at the highest strength and largest elongation.

The mechanical enhancement of the direct spun multi-membrane carbon nanotube yarn by twisting just like tissue paper. A tissue paper consists of flatten $0.2 \mu \mathrm{m}$ thick fiber preferentially aligned along one direction which was taken as a referent to understand the twisting behavior for our membrane fiber. A piece of three layers of single paper with an aspect ratio of $1.5(150 \times 1 \mathrm{~mm})$ and $600 \mu \mathrm{m}$ thick was used corresponding to that of membrane fiber of 1 centimeter. The paper was twisted $25 \mathrm{r} / \mathrm{cm}$ increase the fiber strength of the paper by 3 times from $0.25 \mathrm{MPa}$ to $1 \mathrm{MPa}$, and modulus increased by 8 times from $0.04 \mathrm{MPa}$ to $0.37 \mathrm{MPa}$. When the paper was twisted over 25 $\mathrm{r} / \mathrm{cm}$, it fractures in the middle resulted from the brittleness of the cellulose fiber. The above experiments were conducted by 3 times.

Finally, Poission ratios were measured before and after the twisting. Poission ratio is ratio of the strain across the fiber and that along the fiber. The change of Poisson ratio of the fibers before and after twisting is obtained by measuring the dimensional changes of the fibers from the optical images. The starting fiber has the Poisson ratio in the range of $0.250 \sim 0.265$. Metals have poisson ratio between $0.29 \sim 0.33$ and inorganic solids between $0.2 \sim 0.25$, polymers between $0.13 \sim 0.5$. This value is ten times lower than that of the twisted dry-drawing CNTFs from CNT arrays by twisting which is $2.0 \sim 2.7^{[5]}$.This indicates that our fibers is much denser comparing to their. The density of our fiber is $1.76 \mathrm{~g} / \mathrm{cm}^{3}$, calculated by (Strength $(\mathrm{GPa}) /$ Specific Strength $\left(\mathrm{GPa} /\left(\mathrm{g} / \mathrm{cm}^{3}\right)\right)$, in comparing to their fibers which is $0.8 \mathrm{~g} / \mathrm{cm}^{3[5]}$. The density is $77 \sim 93 \%$ that of graphite $(1.9 \sim$ $2.3 \mathrm{~g} / \mathrm{cm}^{3}$ ). Since our fiber is hollow, the changes of the lateral dimension represent only apparent changes. For a CNT film of mixture of SWNT and MWNT, a negative poison ratio is observed under tensing. As the starting fiber has a hollow membrane structure, the changes of dimensions may partially resulted from the deformation of the hollow, and the Poisson ratio reflects the apparent changes of the dimension of the CNT films on the fiber walls. After twisting at $70 \mathrm{r} / \mathrm{cm}$, the Poisson ratio decreases to $0.165 \sim 0.2$, decreased by ten times. This is the result of the densification of the fibers with reduced voids among the CNTs that reduced the capability of deformation across the fiber. The Poisson ratio of our twisted CNT fiber is lower than those common inorganic solids. The density after densification is $\sim 1.98 \mathrm{~g} / \mathrm{cm}^{3}$.

In summary, twisting of the CVD directly spun membrane fiber remarkably increases the fiber strength, modulus and fractural work. The critical twisting angle is $70 \mathrm{r} / \mathrm{cm}$ where the fiber strength is increased double by the twisting. The modulus increases by 10 times, and the fractural work increases by one time. The Poission ratio if between $0.167 \sim 0.200$ lower than that of the as-spun fiber $(0.250 \sim 0.265)$. Moreover, the electrical conductivity increases to $4.0 \times 10^{5} \mathrm{~S} / \mathrm{m} \sim 30 \%$ higher than that of the starting fiber. The mechanical twisting is simple, which can be performed online to process the CNT fibers and enhance their mechanical and physical properties of the CNT fiber. The critical coefficient is lower than that of traditional fabric indicating CNTF is a new type of fabric.

\section{Experimental}

The fiber was spun by the injection CVD gas flow reaction using acetone as the carbon source dispersed with ferrocene and thiophene. The synthesis conditions are the injection rate of $8 \mathrm{ml} / \mathrm{min}$ and synthesis temperature of $1170 \mathrm{C}$ and hydrogen flow of $1000 \mathrm{sccm}$. The spinning velocity is 5 $\mathrm{m} / \mathrm{min}$. The diameters of the fibers were measured by optical microscope (Olympus BX51M, Tokyo Japan). The surface of the fibers was observed by SEM (XL30E, Phillips Holland).The cross-section of the fibers were obtained by cutting the fibers by electrical sparking at $24 \mathrm{~V}$ into 1 
mm pieces and viewed under SEM. The twisted fibers were tested using tensile micro-fiber tester (XQ-1, Shanghai New Fiber Instruments, China, Shanghai) using gauge length of $10 \mathrm{~mm}$ and tensile speed of $3 \mathrm{~mm} / \mathrm{min}$. The fiber was fixed pneumatic chuck . The Piossion ratio was measured from the optical image of the fibers before and after tensing. The tensing for the measurement of Poisson ratio is performed on high precise fiber tester for short fibers (JSF08, Shanghai Zhongcheng Equipment Co Ltd. Shanghai, China) equipped with a CCD video camera using loading rate of $0.075 \mathrm{~mm} / \mathrm{min}$. The fiber samples were $5 \mathrm{~mm}$ and were fixed on sample holder by dipping epoxy at the two ends of the fiber with the distance of $1 \mathrm{~mm}$, dried in oven at $80 \mathrm{C}$ for $5 \mathrm{~h}$.

\section{Acknowledgements}

Author is gratefu to Prof. Ya-li li at Tianjin University for his carbon nanotube fiber and also indebted to liu yakun for her experiment support

\section{References}

[1] N. K. Nail, and R. Kuchibhotla, "Analytical study of strength and failure behaviour of plain weave fabric composites made of twisted yarns," Composites: Part A, 33 [5], 697-708, (2002).

[2] B. B. Mohamed, and K S. George, "Novel mechanism for spinning continuous twisted composite nanofiber yarns,” European Polymer Journal, 44 [1], 1-12, (2008).

[3] B. Vigolo, A. Penicaud, C. Coulon, C. Sauder, R. Pailler, C. Journet, P. Bernier, and P. Poulin, "Macroscopic fibers and ribbons of oriented carbon nanotubes," Science, 290 [5495], 1331-34, (2000).

[4] K. L. Jiang, Q. Q. Li, and S. S. Fan, "Nanotechnology: spinning continuous carbon nanotube yarns-carbon nanotubes weave their way into a range of imaginative macroscopic applications," Nature, 419 [6909], 801-01, (2002).

[5] M. Zhang, K. R. Atkinson, and R. H. Baughman, "Multifunctional carbon nanotube yarns by downsizing an ancient technology," Science, 306 [5700], 1358-61, (2004).

[6] X. F. Zhang, Q. W. Li, Y. Tu, Y. A. Li, J. Y. Coulter, L. X. Zheng, Y. H. Zhao, Q. X. Jia, D. E. Peterson, and Y. T. Zhu, "Strong carbon-nanotube fibers spun from long carbon-nanotube arrays," Small, 3 [2], 244-48, (2007).

[7] Y. L. Li, I. A. Kinloch, and A. H. Windle, "Direct spinning of carbon nanotube fibers from chemical vapor deposition synthesis,” Science. 304 [5668], 276-78, (2004).

[8] T. W. Cheng, and W. K. Hsu, "Winding of single-walled carbon nanotube ropes: an effective load transfer," Applied Physics Letters, 90 [12], (2007).

[9] N. K. Naik, and V. Madhavan, “Twisted impregnated yarns: elastic properties,” J. Strain Anal. Eng. Des., 35 [2], 83-91, (2000).

[10]Y. Rao, and R. J. Farris, "A modeling and experimental study of the influence of twist on the mechanical properties of high-performance fiber yarns,” J. Appl. Polym. Sci., 77 [9], 1938-49, (2000).

[11]B. Gao, Y. F. Chen, M. S. Fuhrer, D. C. Glattli, and A. Bachtold, "Four-point resistance of individual single-wall carbon nanotubes,” Phys. Rev. Lett., 95 [19], (2005). 